\title{
ASSOCIATION BETWEEN EXPLORATORY ACTIVITY AND SOCIAL INDIVIDUALITY IN GENETICALLY IDENTICAL MICE LIVING IN THE SAME ENRICHED ENVIRONMENT
}

\author{
J. FREUND, ${ }^{\text {a }}$ A. M. BRANDMAIER, ${ }^{\mathrm{b} \dagger}$ L. LEWEJOHANN, ${ }^{\mathrm{c}, \mathrm{d}}$ \\ I. KIRSTE, ${ }^{a}$ M. KRITZLER, ${ }^{e}$ A. KRÜGER, ${ }^{, e}$ N. SACHSER, ${ }^{\circ}$ \\ U. LINDENBERGER ${ }^{\mathrm{b}}$ AND G. KEMPERMANN ${ }^{\mathrm{a}, \mathrm{g*}}$ \\ ${ }^{a}$ CRTD - DFG Research Center for Regenerative \\ Therapies Dresden, Technische Universität Dresden, Germany \\ ${ }^{\mathrm{b}}$ Center for Lifespan Psychology, Max Planck Institute for \\ Human Development, Berlin, Germany \\ ${ }^{\mathrm{c}}$ Department of Behavioral Biology, Westfälische \\ Wilhelms Universität Münster, Germany \\ ${ }^{\mathrm{d}}$ Department of Behavioral Biology, Universität Osnabrück, Germany \\ e Institute for Geoinformatics, Westfälische Wilhelms \\ Universität Münster, Germany \\ f German Research Center for Artificial Intelligence, \\ Saarbrücken, Germany \\ ${ }^{9}$ German Center for Neurodegenerative Disease (DZNE) \\ Dresden, Germany
}

\begin{abstract}
We previously reported that inbred, genetically identical mice living in one enriched environment develop individual behavioral trajectories, indicating increasingly different levels of spatial exploratory behavior as quantified by roaming entropy. Cumulative roaming entropy (cRE) correlated positively with adult hippocampal neurogenesis, a type of plasticity involved in the flexible integration of new information into existing contexts (Freund et al., 2013). The study on which we report here was done in parallel to that first experiment, but here we acquired detailed observational data on the behavior of individual mice. Roaming entropy (RE) was again assessed in real-time with an antenna-based system over the entire experimental period of 3 months. Compared to the least active mice in the enclosure (low number of antenna contacts), the most active animals showed tendencies of increased socially interactive behavior in the final observation block whereas least active mice displayed more self-related behavior (non-social local exploration and play). When looking at roaming behavior, we discovered that RE correlated negatively with latent factors representing social exploratory and non-social exploratory and play behavior. Adult neurogenesis could not be studied in the present cohort but we do know that under identical conditions,
\end{abstract}

\footnotetext{
*Correspondence to: G. Kempermann, German Center for Neurodegenerative Disease (DZNE) Dresden, Germany.

E-mail addresses: gerd.kempermann@dzne.de, gerd.kempermann@crt-dresden.de (G. Kempermann).

+ Equal contribution

* Current address: Brain Imaging and Analysis Center, Duke University Medical Center, Durham, NC, USA.

Abbreviations: cRE, cumulative roaming entropy; CTR, control group; ENR, enriched environment; LA, least active; MA, most active; RE, roaming entropy; RFID, radio-frequency identification.
}

cumulative RE correlated positively with adult hippocampal neurogenesis. We can thus hypothesize that the mice with more exploratory experience in terms of areal coverage (as quantified by RE) and related greater levels of adult hippocampal plasticity, might also be the ones that were less involved in interactions within the group and, hence, more individualistic. While this remains to be confirmed experimentally, the present data suggest that the described mechanism of individualization, which has previously been shown to be hippocampus-dependent, has a social component.

This article is part of a Special Issue entitled: Hippocampus. (c) 2015 IBRO. Published by Elsevier Ltd. All rights reserved.

Key words: hippocampus, dentate gyrus, experience, adult neurogenesis, plasticity, exploration.

\section{INTRODUCTION}

Shemesh and colleagues have reported that mice exposed to an enriched environment during adolescence became more individualistic than under control conditions, in the sense of a weaker dependency between the behavior of individual mice (Shemesh et al., 2013). They drew their conclusion from a detailed analysis of social interactions among the mice based on joint spatial configurations derived from a video-based tracking system. In their discussion, Shemesh et al. highlighted the suggestive relation of their data to our finding that genetically identical mice sharing one enriched environment for a period of 3 months developed stable behavioral trajectories that correlated with adult hippocampal neurogenesis as a measure of structural brain plasticity (Freund et al., 2013).

In the original set-up for the study previously reported by us (Freund et al., 2013), we paralleled two identical experiments, (sharing one control group) the only difference being, that in one enclosure we assessed histological measures of adult neurogenesis at the end of the study, and in the second enclosure we performed a detailed behavioral monitoring. The aim of the behavioral monitoring had been to gain deeper insights into behavioral patterns beyond those assessable by the automated tracking system, for instance, signs of aggressiveness, play behavior, maintenance behavior, and social interaction. We planned to complement the behavioral 
monitoring data with an immunohistochemical follow-up to replicate and broaden our statements about brain-behavior relations; unfortunately, fixation of the mouse brains in the second enclosure failed and all histological data were lost. Although this prevents us from making strong statements about the relationship between more fine-grained behavioral patterns and adult neurogenesis, we do have available the automated tracking data for both environments. From the results in the first enclosure, we also know about the strong correlation between cumulative roaming entropy (cRE) and adult neurogenesis, obtained from the same experimental setup (in particular identical cage layouts, maintenance and handling routines as well as the same staff), the same delivery of animals, and at the same time. We thus analyze the behavioral data set against the backdrop of the neurogenesis data that have already been published (Freund et al., 2013). Based on the data from the second enclosure, we asked the question of how far a large and complex environment would shape individual behavioral profiles in genetically identical mice. Specifically, we intended to learn whether any arising differences in observed behavior would be related to particular RE patterns. We would take any such correlation as the basis for cautious inferences on the relationship between various aspects of behavior and hippocampal neurogenesis, which need to be confirmed eventually in a follow-up study.

\section{EXPERIMENTAL PROCEDURES}

\section{Animals and experimental groups}

We purchased 100 female C57BL/6N mice from as many different litters as possible from a commercial breeder (Charles River, Sulzbach, Germany) at 4 weeks of age; 52 of these were used for the present study. During the first week in our lab, the mice were kept in groups of 10 (see Fig. 1 for the experimental set-up). Within this week, all animals had a radio frequency identification transponder (RFID; Trovan ID-100B Animal Implantable Transponder) implanted in their necks under brief anesthesia with isoflurane. Using marker pens, the mice were marked on ears and tail with an individual color code in order to make them visually distinguishable. The markings were renewed once a week during cage maintenance. The animals were randomly distributed to either the enrichment group (ENR; 40 mice) or the control group (CTR; 12 mice). The randomization was done using a freely accessible computer program for scientific randomizations at www.randomizer.org. In both groups, the animals had bedding, nesting material, and free access to food and water. The animals were kept in a light/dark cycle of $12 \mathrm{~h}$ per phase.

After 105 days in their respective enclosures, ENR and CTR animals were deeply anesthetized with Ketamine and Xylazine $\left(\right.$ Ketanest $^{\circledR}, 100 \mathrm{mg} / \mathrm{kg}$ body weight and Rompun $^{\circledR}, 10 \mathrm{mg} / \mathrm{kg}$ body weight in saline solution). This was followed by a transcardial perfusion with $0.9 \% \mathrm{NaCl}$ and subsequently with $4 \%$ paraformaldehyde $\left(\right.$ Roti $^{\circledR}$ Histofix 4\%, Carl Roth $\mathrm{GmbH}+$ Co. KG, Karlsruhe, Germany). The brains were dissected below the brainstem and subsequently wet-weighed. Three weeks before, all animals received three injections of BrdU (5bromo-2'-deoxyuridine; SIGMA-Aldrich; conc.: $50 \mathrm{mg} / \mathrm{kg}$ body weight) intraperitoneally, one on each of three consecutive days. Because of a fixation failure, antigens were not appropriately preserved in the tissue samples and cell genesis could not be further explored in this study.

In all experimental steps, we strictly adhered to national laws and institutional guidelines, and all experiments were approved in advance by the appropriate authorities at Westfälische Wilhelms-Universität Münster (Reference No. 8.87-50.10.36.08.250).

\section{Enclosures and tracking system}

40 ENR animals lived in an enclosure with a square ground area of $1.75 \mathrm{~m}$ side length, $2 \mathrm{~m}$ height, and a total area of approximately $5 \mathrm{~m}^{2}$. The enclosure's interior and positions of the antennas were identical to the enclosure we described before (Freund et al., 2013): The basic setup featured two ground levels and three elevated levels through which the animals could move freely. Water and food sources were available at each level. All levels were connected with plastic tubes. Two nesting boxes were provided and various enrichment objects were placed in the enclosure (e.g., plastic and cardboard tubes, wooden scaffold, flower pots). The mice could leave the enclosure, for example, in the event of high social pressure, by crossing a tube in the enclosure's front leading to a water basin. After crossing the water, they could enter an emigration cage.

Data were collected using the software program Jerry 2 Recorder, developed for these purposes by the Institute for Geoinformatics in Münster, and stored using a MySQL database (Kritzler et al., 2006).

Twenty plastic rings with RFID antennas were installed throughout the enrichment enclosure, recording

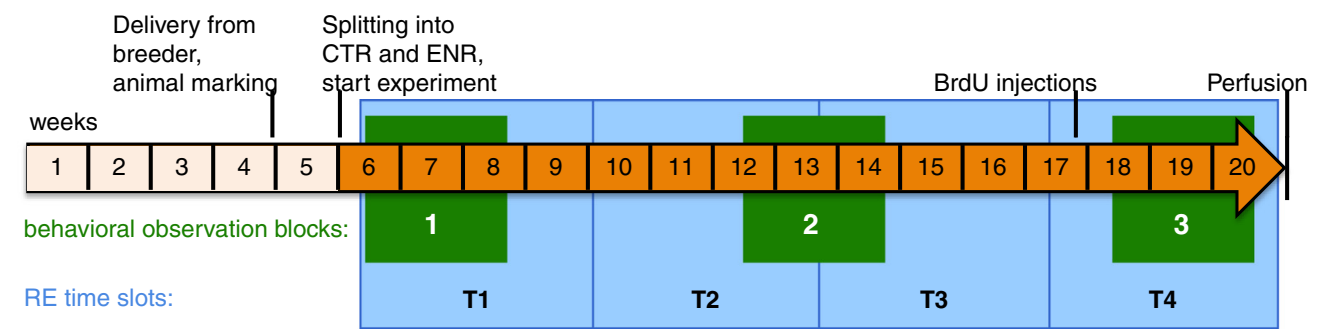

Fig. 1. Experimental schedule (see text for details). 
detailed information on the animals' movements in the cage as well as the use of nesting boxes and water sources. CTR was housed in three standard cages (size 3) with four animals inhabiting each. CTR cages were all equipped with a single RFID antenna. For an overview of the enclosure's layout as well as details on cage maintenance procedures, see Freund et al. (2013).

\section{Behavioral observations}

To manually assess behavior of the mice, we employed a focal animal sampling and continuous recording method. Behavioral measures were either recorded as frequencies of events or duration of states (see Table 1). Behavioral monitoring was performed in three blocks of 12 days in order to assess spontaneous behavior of the animals in greater detail (Fig. 1). In each block, the behavior of 24 focus animals (out of the entire cohort of 40) was recorded. Each focus animal was observed in six sessions lasting up to $5 \mathrm{~min}$ each, resulting in a total of up to $30-\mathrm{min}$ observation time per focus animal and observation block. No animal was observed more than once per day. The focus animals were determined by the mice's activity in the week preceding the respective observation block. Using the RFID recordings, the total number of antenna contacts in that week was calculated. The 12 most active (MA) and the 12 least active (LA) animals were designated as focus animals for the upcoming observational block. In a preliminary experiment using different mice, key behaviors of female mice in this environment had been determined. All observed behaviors were defined in detail in an extensive ethogram (see Table 1 for a list and short definitions of recorded behaviors) so that the frequencies and durations could be recorded and

Table 1. List of recorded behaviors; $f=$ frequency of behavior recorded, $d=$ duration of behavior recorded, FA $=$ focus animal

\begin{tabular}{|c|c|c|}
\hline \multirow[t]{3}{*}{$\begin{array}{l}\text { Social } \\
\text { behaviors }\end{array}$} & Socio-positive behavior & $\begin{array}{l}\text { Body contact }(f) \text { - FA touches the body of conspecific with any part of its body except teeth or } \\
\text { nose } \\
\text { Allogroom }(f) \text { - FA scratches, paws or licks the body of another mouse }\end{array}$ \\
\hline & $\begin{array}{l}\text { Social exploratory } \\
\text { behavior }\end{array}$ & $\begin{array}{l}\text { Naso-nasal sniff }(f) \text { - FA positions twitching tip of its snout within one head length of another } \\
\text { mouse's nose } \\
\text { Anogenital sniff }(f) \text { - FA positions twitching tip of its snout below the root of another mouse's tail } \\
\text { Approach }(f) \text { - FA moves with its snout directed toward another mouse until both are at least } \\
\text { within one body length } \\
\text { Is approached }(f) \text { - Mouse approaches FA until both are at least within one body length }\end{array}$ \\
\hline & Agonistic behavior & $\begin{array}{l}\text { Chase }(f) \text { - FA runs after another mouse and may lunge at it } \\
\text { Is chased }(f)-\text { A mouse runs after FA and may lunge at it } \\
\text { Push }(f) \text { - FA pushes conspecific with its forepaws while both are in a tube } \\
\text { Receive push }(f) \text { - FA is pushed by another mouse while in a tube } \\
\text { Bite }(f) \text { - FA drives its teeth into fur, skin or tail of another mouse } \\
\text { Receive bite }(f) \text { - A mouse drives its teeth into fur, skin or tail of FA } \\
\text { Fight }(f) \text { - FA struggles with another mouse in physical contact. They may show additional } \\
\text { agonistic behavior patterns } \\
\text { Retreat }(f) \text { - FA moves directionally away from a conspecific that has approached it in the } \\
\text { preceding } 5 \mathrm{~s} \\
\text { Tail tremble }(f) \text { - Tail of FA performs fast vibrating movements. The rest of the body does not } \\
\text { move }\end{array}$ \\
\hline
\end{tabular}

$\begin{array}{cl}\begin{array}{c}\text { Self-related } \\ \text { behaviors }\end{array} & \begin{array}{l}\text { Non-social exploratory and } \\ \text { play behavior }\end{array}\end{array}$ forepaws neither have tactile contact with a part of the enclosure nor a conspecific Dig $(f)$ - FA moves bedding along its underside with forepaws and subsequently kicks it backward with hindpaws

Sprint $(f)$ - FA suddenly moves forward fast for at least one body length and then stands still Rear with support $(f)-F A$ raises itself on hindpaws, stretches its snout into the air while supporting itself with one or both forepaws on any part of the enclosure but not a conspecific Manipulate object $(f)$ - FA gnaws at an object, drags or pushes it with its head or paws Leap $(f)$ - FA jumps forward on a level surface for at least one body length. The forepaws reach the surface before the hindpaws

Drink $(f+d)-$ FA touches a source of water with its snout and licks it

Feed $(f+d)-$ FA gnaws at a piece of food and ingests part of it

Root $(f+d)-$ FA has its snout lowered in the bedding and pushes it aside with its forepaws Autogroom $(f+d)-$ FA scratches, paws or licks its own body

Nesting $(f+d)-$ FA breaks up soft material (paper, fabric, bedding etc.) and carries it to the nesting space and/or arranges the material with its snout and forepaws to form a small hollow Rest $(f+d)-$ FA has not moved for at least $10 \mathrm{~s}$ (duration is subtracted from the observation time)

Stereotypic behavior
Stereotypic behavior $(f)$ - FA compulsively repeats a behavior without obvious goal or function (e.g., repetitive jumping, repetitive climbing in a circular motion or overgrooming) 
Table 2. Behavioral statistics for observation blocks 2 and 3 : comparison between most active (MA) and least active (LA) animals (Mann-Whitney $U$ test), medians for MA and LA animals, $U$ - and $p$-values (uncorrected). The following items were never observed and thus omitted from the table: bite, receive bite, fight, chase and stereotypic behavior. Gray cells $=$ behavioral categories, $f=$ frequencies and $d=$ durations of behavior

\begin{tabular}{|c|c|c|c|c|c|c|c|c|c|}
\hline & \multirow{2}{*}{ Behavior } & \multicolumn{4}{|c|}{ Block 2} & \multicolumn{4}{|c|}{ Block 3} \\
\hline & & $\begin{array}{c}\text { media } \\
n \text { LA }\end{array}$ & $\begin{array}{c}\text { media } \\
\text { n MA }\end{array}$ & U & p & $\begin{array}{c}\text { media } \\
n \text { LA }\end{array}$ & $\begin{array}{l}\text { media } \\
\text { n MA }\end{array}$ & U & p \\
\hline \multirow{14}{*}{$\begin{array}{l}\text { Social } \\
\text { Behaviors }\end{array}$} & Body contact (f) & 8.00 & 7.50 & 67.5 & 0.817 & 9.50 & 12.00 & 105.5 & 0.056 \\
\hline & Allogroom (f) & 0.50 & 0.50 & 68.5 & 0.853 & 0.50 & 1.50 & 92.0 & 0.239 \\
\hline & Socio-positive Behavior (f) & 9.50 & 9.50 & 69.0 & 0.885 & 10.50 & 15.50 & 105.5 & 0.056 \\
\hline & Naso nasal sniff (f) & 1.00 & 0.50 & 70.5 & 0.952 & 1.00 & 0.50 & 53.0 & 0.257 \\
\hline & Anogenital sniff (f) & 0.00 & 0.00 & 71.0 & 0.972 & 0.00 & 0.00 & 55.5 & 0.267 \\
\hline & Approach (f) & 6.00 & 4.00 & 60.0 & 0.504 & 8.50 & 6.00 & 49.0 & 0.192 \\
\hline & Is approached (f) & 9.00 & 7.00 & 74.5 & 0.908 & 7.00 & 8.50 & 90.0 & 0.310 \\
\hline & $\begin{array}{l}\text { Social Exploratory Behavior } \\
\text { (f) }\end{array}$ & 16.00 & 13.50 & 71.5 & 1.000 & $\mid 17.50$ & $\mid 17.50$ & 65.5 & 0.728 \\
\hline & Is chased (f) & 0.00 & 0.00 & 78.0 & 0.359 & 0.00 & 0.00 & 72.0 & 1.000 \\
\hline & Push (f) & 0.50 & 0.50 & 78.0 & 0.723 & 0.50 & 0.50 & 69.0 & 0.875 \\
\hline & Receive push (f) & 1.00 & 0.50 & 71.5 & 1.000 & 0.00 & 1.50 & 102.0 & 0.061 \\
\hline & Retreat (f) & 0.00 & 0.00 & 72.0 & 1.000 & 0.00 & 0.00 & 72.0 & 1.000 \\
\hline & Tail tremble (f) & 0.00 & 1.00 & 91.0 & 0.230 & 1.00 & 1.50 & 87.0 & 0.387 \\
\hline & Agonistic Behavior (f) & 1.00 & 2.50 & 95.0 & 0.182 & 2.50 & 2.50 & 81.0 & 0.617 \\
\hline
\end{tabular}

\begin{tabular}{|c|c|c|c|c|c|c|c|c|c|}
\hline \multirow{8}{*}{$\begin{array}{l}\text { Self-related } \\
\text { Behaviors }\end{array}$} & Rear independently (f) & 7.50 & 3.00 & 20.5 & 0.003 & 6.00 & 3.00 & 52.0 & 0.257 \\
\hline & $\operatorname{Dig}(\mathrm{f})$ & 0.00 & 0.00 & 77.0 & 0.714 & 0.00 & 0.00 & 62.0 & 0.471 \\
\hline & Sprint (f) & 5.00 & 4.00 & 57.0 & 0.399 & 5.00 & 2.50 & 27.0 & 0.010 \\
\hline & Rear supportedly (f) & 6.00 & 3.00 & 36.5 & 0.042 & 5.50 & 4.00 & 43.0 & 0.097 \\
\hline & Manipulate object (f) & 8.50 & 4.00 & 36.0 & 0.039 & 5.50 & 5.00 & 54.5 & 0.323 \\
\hline & Leap (f) & 10.50 & 2.00 & 26.0 & 0.008 & 9.00 & 3.50 & 51.5 & 0.247 \\
\hline & $\begin{array}{l}\text { Non-social Exploratory and } \\
\text { Play Behavior (f) }\end{array}$ & 36.50 & 19.00 & 15.0 & 0.001 & 34.50 & 19.50 & 35.5 & 0.037 \\
\hline & Drink (f) & 3.00 & 3.50 & 66.5 & 0.771 & 4.00 & 2.00 & 38.5 & 0.054 \\
\hline & Drink (d) & 37.50 & 35.00 & 66.0 & 0.751 & 41.00 & 32.50 & 62.0 & 0.583 \\
\hline & Feed (f) & 10.50 & 7.00 & 54.5 & 0.326 & 14.00 & 11.50 & 52.5 & 0.271 \\
\hline & Feed $(d)$ & $\begin{array}{r}576.0 \\
0\end{array}$ & $\begin{array}{r}168.0 \\
0\end{array}$ & 30.5 & 0.018 & $\begin{array}{r}595.5 \\
0 \\
\end{array}$ & $\begin{array}{r}455.0 \\
0 \\
\end{array}$ & 54.0 & 0.319 \\
\hline & Root (f) & 8.00 & 6.50 & 54.0 & 0.310 & 12.00 & 9.50 & 48.0 & 0.173 \\
\hline & Root (d) & $\begin{array}{r}101.5 \\
0 \\
\end{array}$ & $\begin{array}{r}126.0 \\
0 \\
\end{array}$ & 73.0 & 0.977 & $\begin{array}{r}162.0 \\
0 \\
\end{array}$ & 84.00 & 56.0 & 0.371 \\
\hline & Nesting (f) & 0.00 & 0.00 & 62.5 & 0.533 & 1.00 & 0.50 & 64.0 & 0.646 \\
\hline & Nesting (d) & 0.00 & 0.00 & 60.5 & 0.449 & 31.00 & 1.50 & 61.0 & 0.524 \\
\hline & Autogroom (f) & 10.00 & 5.50 & 41.0 & 0.077 & 9.00 & 8.50 & 71.0 & 0.977 \\
\hline & Autogroom (d) & $\begin{array}{r}180.5 \\
0\end{array}$ & $\begin{array}{r}182.0 \\
0\end{array}$ & 75.5 & 0.862 & $\begin{array}{r}172.0 \\
0\end{array}$ & $\begin{array}{r}138.5 \\
0\end{array}$ & 68.5 & 0.862 \\
\hline & Rest (f) & 0.00 & 0.00 & 74.0 & 0.916 & 0.00 & 0.00 & 71.0 & 0.964 \\
\hline & Rest (d) & 0.00 & 0.00 & 74.0 & 0.918 & 0.00 & 0.00 & 70.0 & 0.894 \\
\hline & Maintenance Behavior (f) & 32.00 & 24.50 & 51.0 & 0.236 & 41.00 & 32.50 & 41.0 & 0.078 \\
\hline & Maintenance Behavior (d) & $\begin{array}{r}957.0 \\
0\end{array}$ & $\begin{array}{r}769.5 \\
0\end{array}$ & 49.0 & 0.198 & $\begin{array}{r}1020 . \\
50\end{array}$ & $\begin{array}{r}856.5 \\
0\end{array}$ & 36.0 & 0.039 \\
\hline
\end{tabular}

compared. Unless otherwise stated in Table 1, events were counted again after the distance between the animals had exceeded one body length in case of social behaviors and/or if the same behavior was carried out after an interruption of at least $3 \mathrm{~s}$. If the focus animal moved into an area where it could not be watched or recognized by the observer, e.g., due to insufficient light or possible confusion with conspecifics, "no visual contact (duration)" was recorded. During this time, no precise statement about FA's behavior could be made and the duration was thus subtracted from the observation time. The ethogram was checked for completeness by matching it against other ethograms developed and applied in the Department of Behavioral Biology (e.g., Marashi et al., 2003; Lewejohann et al., 2009a,b) and sample ethograms at www.mousebehavior.org. The resulting list of recorded behaviors comprises social and self-related behaviors in five broader behavioral categories: Socio-positive and agonistic behavior, social and non-social exploration and play behavior, and maintenance (see Table 1 for the recorded behavior items). Since frequency and duration results of the same 
Table 3. Statistics for all three observation blocks: correlation between recorded behavior and cRE at T4 (Pearson correlation), Pearson's $r$ and $p$ values (uncorrected). The following items were never observed and thus omitted from the table: bite, receive bite, fight, chase and stereotypic behavior. Gray cells $=$ behavioral categories, $f=$ frequencies and $d=$ durations of behavior

\begin{tabular}{|c|c|c|c|c|c|c|c|}
\hline & \multirow{2}{*}{ Behavior } & \multicolumn{2}{|c|}{ Block 1} & \multicolumn{2}{|c|}{ Block 2} & \multicolumn{2}{|c|}{ Block 3} \\
\hline & & $\mathbf{r}$ & $\mathrm{p}$ & $\mathbf{r}$ & $\mathbf{p}$ & $\mathbf{r}$ & $\mathbf{p}$ \\
\hline \multirow{14}{*}{$\begin{array}{c}\text { Social } \\
\text { behaviours }\end{array}$} & Body contact (f) & 0.186 & 0.385 & -0.503 & 0.012 & -0.125 & 0.562 \\
\hline & Allogroom (f) & 0.091 & 0.673 & -0.198 & 0.354 & 0.111 & 0.604 \\
\hline & Socio-positive Behavior (f) & 0.147 & 0.494 & -0.488 & 0.016 & -0.027 & 0.901 \\
\hline & Naso nasal sniff (f) & 0.437 & 0.033 & -0.626 & 0.001 & -0.147 & 0.494 \\
\hline & Anogenital sniff (f) & 0.470 & 0.020 & -0.412 & 0.046 & -0.485 & 0.016 \\
\hline & Approach (f) & 0.239 & 0.260 & -0.444 & 0.030 & -0.229 & 0.283 \\
\hline & Is approached (f) & 0.286 & 0.176 & -0.381 & 0.067 & 0.124 & 0.564 \\
\hline & Social Exploratory Behavior (f) & 0.387 & 0.062 & -0.487 & 0.016 & -0.114 & 0.595 \\
\hline & Is chased $(\mathrm{f})$ & 0.216 & 0.311 & -0.008 & 0.969 & 0.175 & 0.412 \\
\hline & Push (f) & 0.115 & 0.591 & -0.079 & 0.713 & -0.033 & 0.878 \\
\hline & Receive push (f) & 0.082 & 0.704 & -0.438 & 0.032 & -0.099 & 0.646 \\
\hline & Retreat $(\mathrm{f})$ & -0.060 & 0.780 & 0.256 & 0.226 & 0.252 & 0.235 \\
\hline & Tail tremble (f) & -0.346 & 0.097 & -0.252 & 0.234 & 0.106 & 0.624 \\
\hline & Agonistic Behavior (f) & $\begin{array}{l}-0.251 \\
\end{array}$ & 0.236 & -0.100 & 0.643 & 0.135 & 0.529 \\
\hline
\end{tabular}

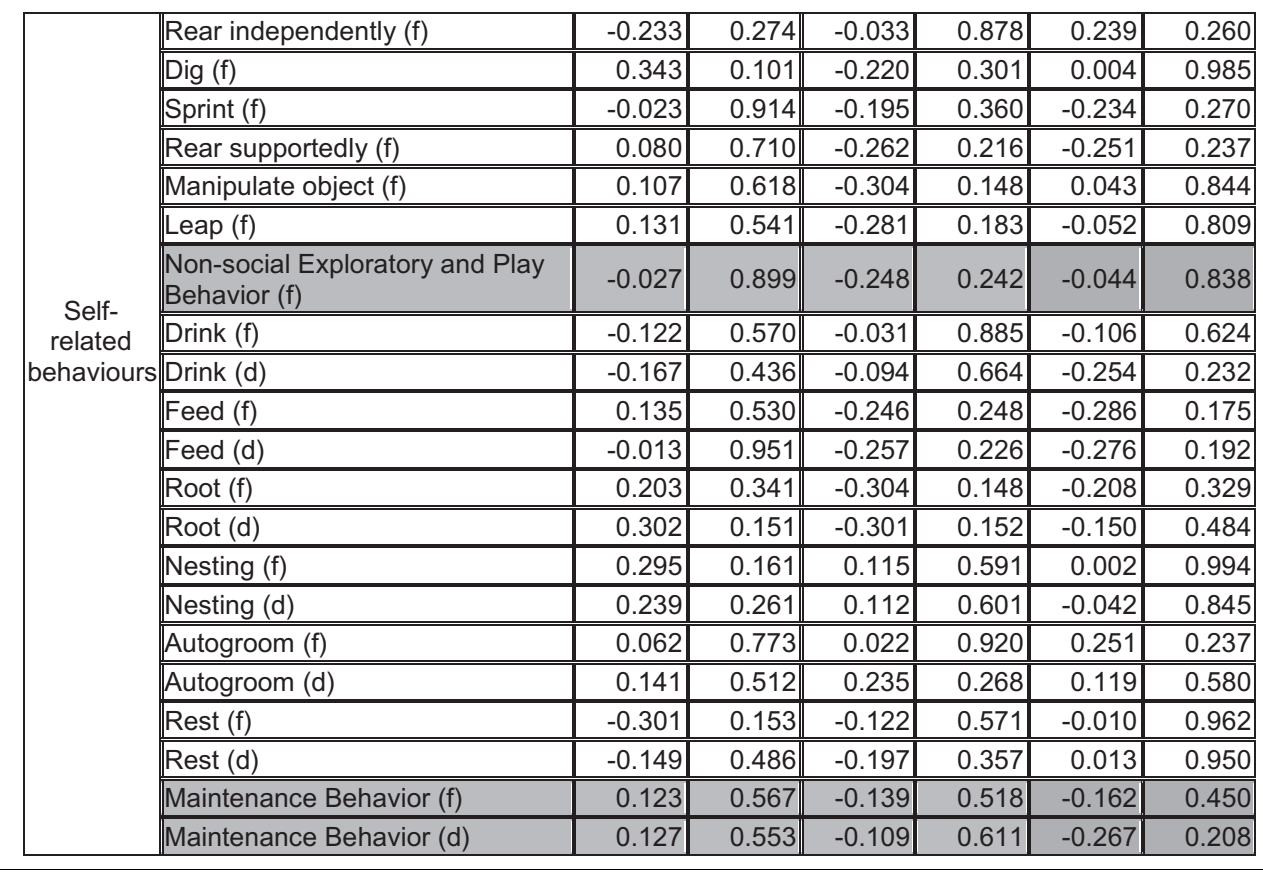

behavioral pattern are generally consistent with each other, later stages of data analysis focused on only frequency for all items (Terranova and Laviola, 2005).

A portable computer with animal observation software (The Observer ${ }^{\circledR}$ XT 7.0, Noldus Information Technology, Wageningen, The Netherlands) was used to record and store the sessions.

\section{Data collection and aggregation}

Each time a mouse entered the tuning range of an antenna, a data base entry was created recording time and date, mouse identifier, and antenna identifier. In the course of the experiment, the RFID system recorded more than $7.6 \mathrm{M}$ events. For a quantitative analysis of this tremendous amount of information, we first performed a data reduction. Because the natural activity period of mice is the nighttime, only night segments (artificial dark phases from $8 \mathrm{pm}$ to $8 \mathrm{am}$ ) were taken into account. In a second step, we calculated discrete time-series of antenna contacts for each mouse and each night segment with 8640 elements of $5 \mathrm{~s}$ each.

The observer (J.F.) kept a manual log file over the entire time of the experiment, particularly recording all events that could possibly have disturbed the mice or interfered with the data collection (e.g., a person entering the cage for cleaning or maintenance, computer reboots, and antenna failures). All days, on which such computer-related failures were recorded, were not included into the analysis of roaming entropy (RE, see next section). Any 
Table 4. Estimated factor loadings for a Poisson factor model of count data from behavioral observations. Two-tailed $p$-values correspond to null hypotheses of zero loadings

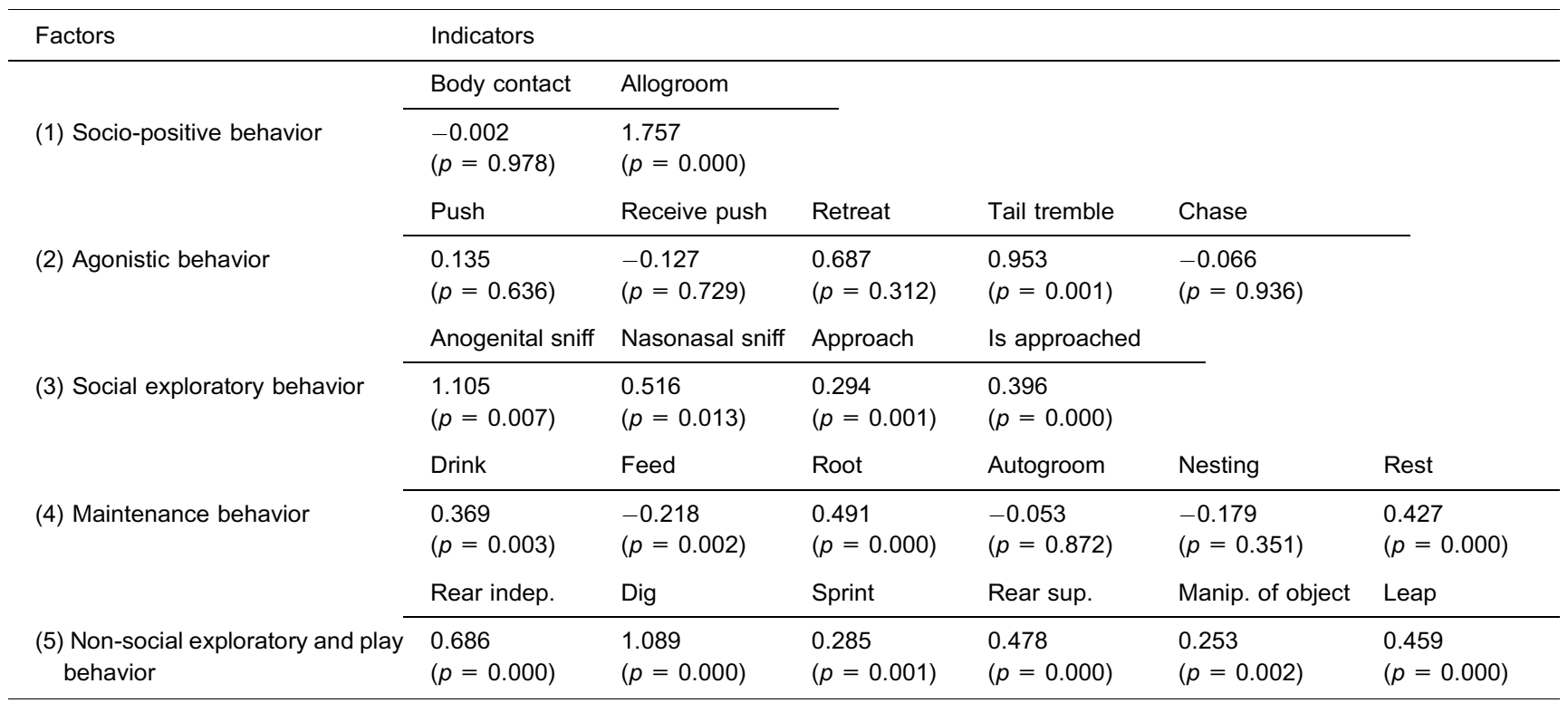

day, on which persons interfered with the mice, and the day thereafter, were excluded to allow for more stable estimates of $\mathrm{CRE}$, but were not excluded when RE was correlated with ethogram-based observations. Thus, 91, respectively, 64 of 96 total observation days were taken into account for our subsequent analyses.

\section{RE}

We have developed RE as an ethologically valid index for spatial (global) exploratory behavior (Freund et al., 2013). To avoid confusion with the local social and self-related exploration behavior recorded in the live observations, the term "exploration" will be used for the live observation data in this paper, while global spatial exploration will be referred to exclusively as "RE".

Briefly, RE is the entropy of the probability distribution of finding a mouse at a given antenna at a given time and, thus, it is an indicator of the territorial range covered by a given mouse in a given period of time. RE will be low for a mouse with a stable and limited home range, but, importantly, independent of the amount of locomotion within that area. On the other hand, a large territory might still be covered with relatively low RE, if a low number of stable sites of attendance are spread out over a larger area. In contrast, RE will be high, if the territorial coverage is more evenly distributed over the entire area (see also Supplemental movies S1 and S2 in Freund et al., 2013) and our online simulation of RE (http://brandmaier.de/roamingentropy/).

Based on the observed frequencies in our observed time series, the probabilities, $p_{i, j, t}$, of a mouse $i$ being at antenna $j$ at day $t$ were estimated. In analogy to the Shannon entropy, we calculated the RE, that is, the entropy of the roaming distribution of mouse $i$ at day $t$ :

$\mathrm{RE}_{i, t}=-\sum_{j=l}^{k}\left(p_{i, j, t} \log p_{i, j, t}\right) / \log (k)$ with $k$ being the number of antennas in the cage $(k=20)$. The RE values range between 0 and 1 .

Analogous to (Freund et al., 2013), measurements of RE were aggregated into four adjacent time periods (T1, T2, T3, and T4), each representing the average RE over 24 calendar days, and summed over time periods to obtain an index of "cumulative roaming entropy" (cRE; i.e., $\quad c R E_{\mathrm{T} 1}=\mathrm{RE}_{\mathrm{T} 1} ; \quad \mathrm{cRE}_{\mathrm{T} 2}=\mathrm{cRE}_{\mathrm{T} 1}+\mathrm{RE}_{\mathrm{T} 2} ; \quad \mathrm{cRE}_{\mathrm{T} 3}=$ $\left.\mathrm{cRE}_{\mathrm{T} 2}+\mathrm{RE}_{\mathrm{T} 3} ; \mathrm{cRE}_{\mathrm{T} 4}=\mathrm{cRE}_{\mathrm{T} 3}+\mathrm{RE}_{\mathrm{T} 4}\right)$.

\section{Statistics}

We tested for differences in physiological measures (body and brain weights) as well as for differences in total activity (total antenna contacts) between ENR and CTR animals using Student's $t$-test (two-tailed, unpaired). Differences in variance in body/brain weight ratios between the two groups were assessed using a twosided $F$-test.

Spearman rank correlation $\rho$ (two-sided) was used to examine the correlation of $\mathrm{CRE}$ at T4 with the number of antenna contacts of the ENR mice. Further, the variances in $\mathrm{CRE}$ at both $\mathrm{T} 1$ and $\mathrm{T} 4$ were compared between the ENR animals reported on in the present paper and those of our previous report (Freund et al., 2013) using a two-sided F-test.

Observational behavior data were recorded from subsets of 24 ENR animals (see above) in each of the three observation blocks. Counts and - if available durations of each of the behavioral items defined in the ethogram were compared between the MA and LA group using Mann-Whitney-U-test (two-sided, unpaired). All the available behavioral data were furthermore tested for a correlation to the animals' cRE values at T4 (Pearson correlation, two-sided).

Eighteen of the 24 animals observed in blocks 2 and 3 , respectively, were focus animals in both blocks. Therefore, a combined analysis of the two later blocks 
Table 5. Correlation coefficients and $p$-values of single degree-offreedom Wald tests against zero correlation based on latent variable modeling. The bold values are statistically significant at $p<0.05$

\begin{tabular}{llll}
\hline \multirow{2}{*}{ Factors } & \multicolumn{3}{l}{ Roaming entropy } \\
& T2 \& T3 & T2 & T3 \\
\hline Socio-positive & 0.066 & -0.091 & 0.312 \\
& $(p=0.751)$ & $(p=0.727)$ & $(p=0.313)$ \\
Agonistic & -0.124 & -0.172 & -0.108 \\
& $(p=0.632)$ & $(p=0.742)$ & $(p=0.737)$ \\
Social expl. & $-\mathbf{0 . 4 3 6}$ & $-\mathbf{0 . 5 0 8}$ & -0.253 \\
& $(p=0.010)$ & $(p=0.005)$ & $(p=0.459)$ \\
Maintenance & -0.316 & 0.033 & $-\mathbf{0 . 6 7 9}$ \\
& $(p=0.068)$ & $(p=0.897)$ & $(p=0.000)$ \\
Non-soc. & $-\mathbf{0 . 4 4 6}$ & -0.236 & $-\mathbf{0 . 7 1 3}$ \\
expl. + Play & $(p=0.003)$ & $(p=0.287)$ & $(p=0.000)$ \\
\hline
\end{tabular}

was possible using the data obtained from these 18 mice. We used them for the factor analysis described in Table 4 and the subsequent average RE correlation with behavioral factors (Wald test, results in Table 5).

\section{RESULTS}

\section{Brain and body weight}

A weekly weight check confirmed that all animals showed a normal age-dependent increase in body weight over time (Fig. 2A). Body weight did not differ between ENR and CTR at the beginning of the experiment $(t=-0.066, \quad d f=18.732, \quad p=0.948)$. ENR animals tended to be slightly heavier than CTR in the final measurement but the difference was not statistically significant $(t=-1.771, d f=25.554, p=0.088$; $t$-tests, two-tailed, unpaired).

In contrast to the body weights, brain weights could only be evaluated at the end of the experiment (Fig. 2B). There was a non-significant trend for lighter brains in ENR $(t=-1.9115, d f=38.828, p=0.064$; $t$-test, two-tailed, unpaired).

The ratio of body to brain weight showed a higher variance in the ENR group compared to the control ( $F=0.304, p=0.038, F$-test, two-sided).

\section{RE decreased over the time of the experiment, but variance in RE increased}

As expected, the obtained antenna data showed that the ENR animals displayed significantly more overall activity than CTR (total number of antenna contacts; ENR vs. CTR: $t=22.475, d f=49.809, p=2.20 \times 10^{-16}$, see Fig. 2C). There was also no difference in total antenna contacts between ENR and the analogous data from (Freund et al., 2013) (comparison between the two
A
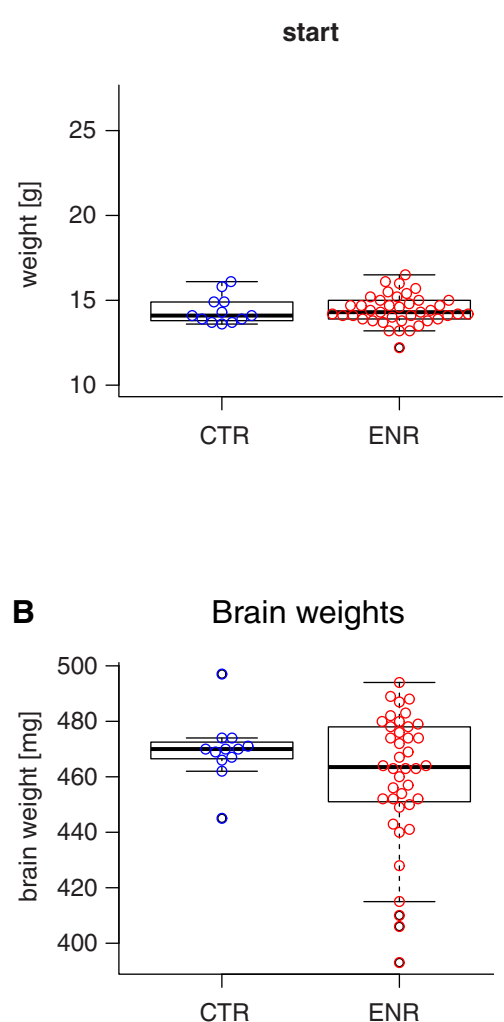

Body weights

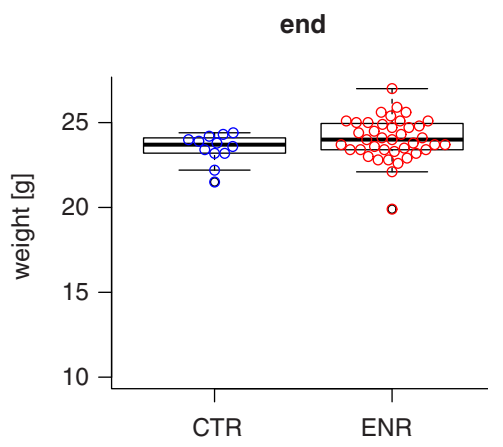

C Antenna contacts

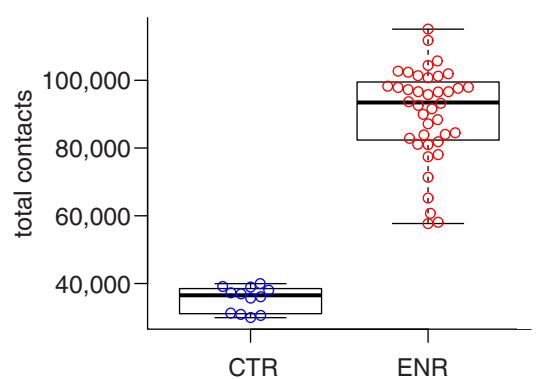

Fig. 2. Weights and raw antenna contacts: (A) body weight development: Body weights of CTR and ENR animals at the first (left) and final measurement, i.e., at the day of perfusion. Circles show each measurement point; (B) brain weights of CTR and ENR at the time of perfusion; (C) total number of antenna contacts over the experimental period of 3 months. The greater number of antenna contacts in the ENR condition not only reflects greater activity, but also the larger cage size and is also influenced by the greater number of antennas. An important detail is the spread among the ENR animals (blue = control group, red = enrichment group). 
enrichment enclosures: $t=-0.4714, \quad d f=65.416$, $p=0.639, t$-tests, two-tailed, unpaired).

A first descriptive analysis of the RE revealed that the nightly mean slightly decreased over the course of the experiment (Fig. 3A). The animals showed individual differences in their RE patterns (see two extreme examples in Fig. $3 \mathrm{C}$ for further illustration of the RE measure). This was associated with an increase in variance in nightly RE (Fig. 3B).

This implies that over time the mice tended to roam the enclosure less, even though the decrease was rather shallow. From the non-automated behavioral monitoring, there were no general signs of strong habituation. At the same time, however, the variance in roaming behavior increased (Fig. 3B). Also note, that in contrast to the overall tendency, the variance was comparatively high in the very first few days, massively decreasing within only a week only to pick up slowly over the remaining weeks. The fact that mean RE values decreased while their variances increased at the same time implies that per actual amount of roaming at a given time, the animals became increasingly more different over the course of the 3-month study period.

\section{CRE}

This behavioral pattern can also be presented in a different format that reveals additional insight. In order to account for the cumulative nature of experience, the derived measure of cRE was calculated (Freund et al., 2013).

\section{A \\ RE nightly means}

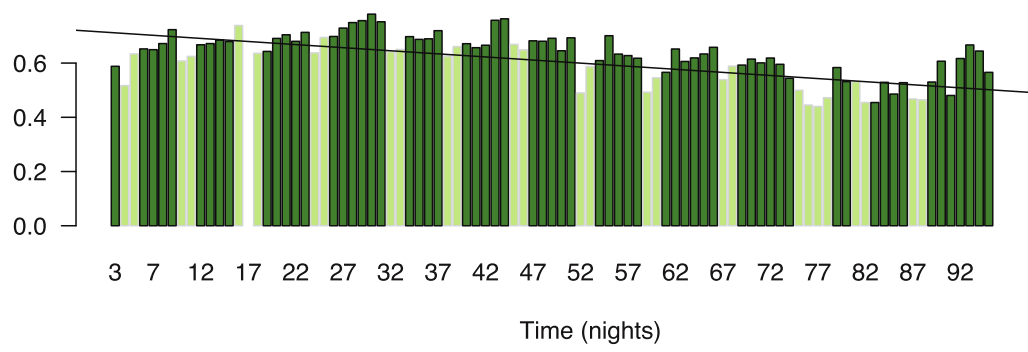

B RE nightly variances

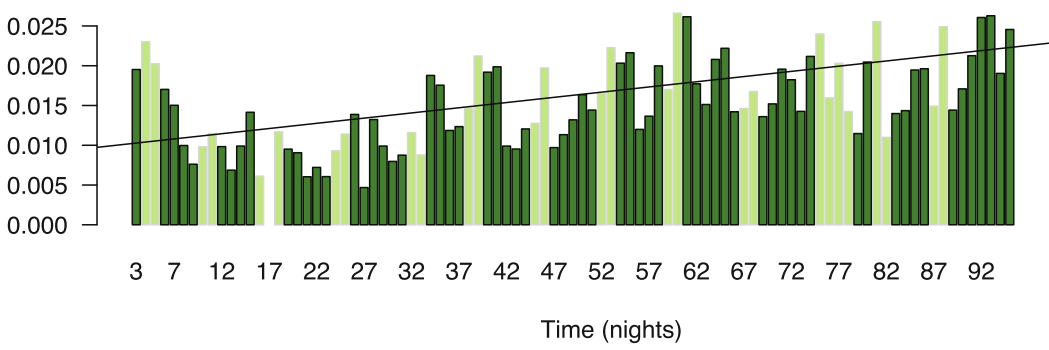

C

Animal \# 30

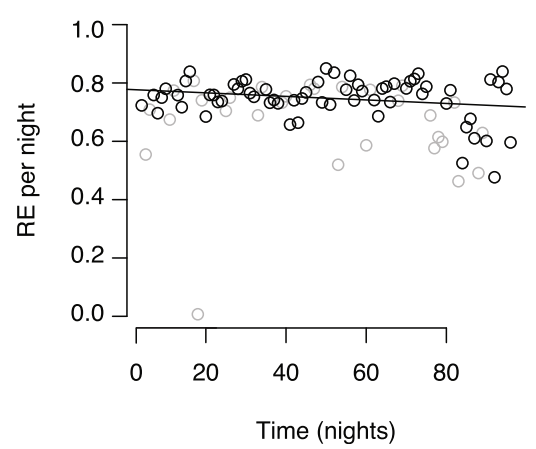

Animal \# 51

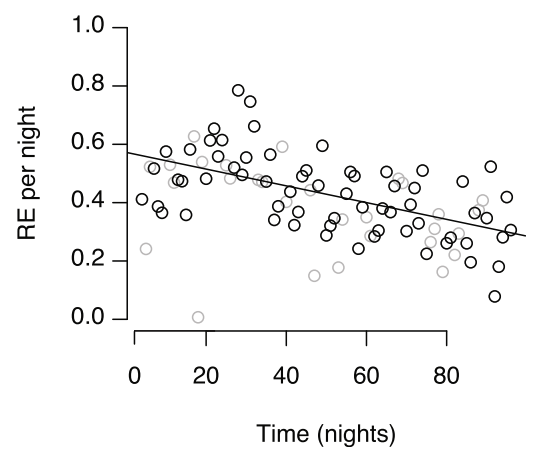

Fig. 3. Nightly RE values in ENR: (A) mean decreased over time: slope of trendline: $m=-0.405$; (B) variance in nightly RE values increased over time: slope of trendline: $m=0.480$ (raw data before cleaning, missing value in night 17 due to a system failure); (C) two examples of nightly RE development: mouse \#30 showed relatively stable RE values within a narrow range while mouse \#51 showed decreasing RE values with high fluctuation; Nights that were excluded from the final analysis are shown as light-green bars in (A) and (B) and as light-gray circles in (C) (see text for details). 

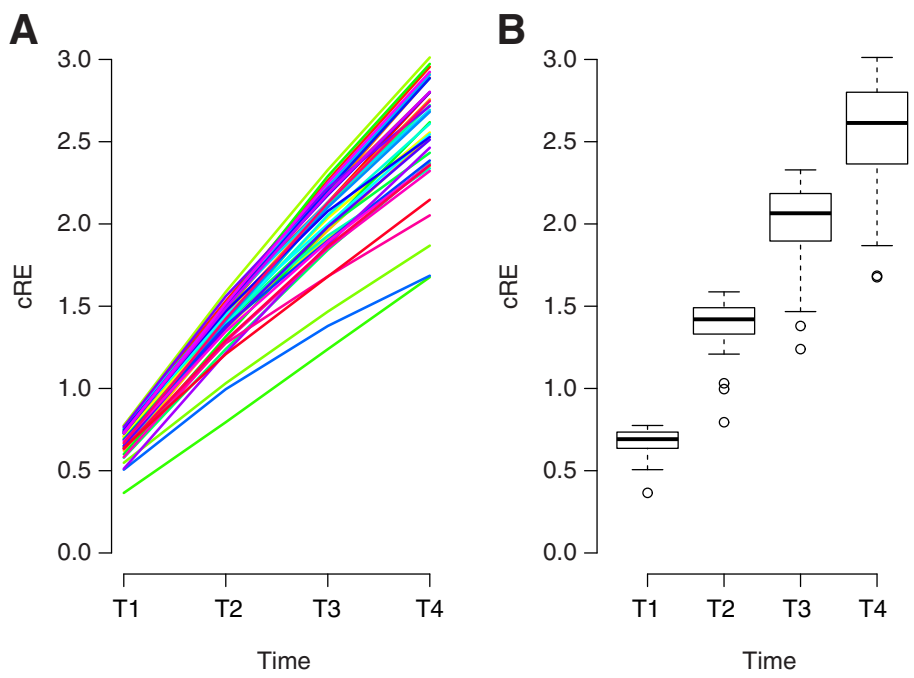

Fig. 4. CRE development over time. (A) Line plot, in which each line represents one mouse (colors were used only to facilitate the distinction of individual lines; (B) same data in a boxplot for each of the four time-points.

cRE takes into account the animal's fluctuations in RE, while still visualizing the direction its development takes in relation to the other individuals over time. We found a fan-like pattern of divergence in cRE between the different animals, highlighting the fact that the mice develop stable behavioral trajectories (with respect to roaming behavior, see Fig. 4). cRE at T4 did not correlate with the total number of antenna contacts $(\rho=0.128, \quad p=0.429$, Spearman's rank correlation $\rho$ ).

Taken together these data draw a pattern that is essentially identical to the picture reported in Ref. Freund et al. (2013). However, note that variances differed between cages at T1, $F(1,76)=8.953, p=0.004$, as well as at T4, $F(1,76)=7.670, p=0.007$ (F-test, two-sided).

\section{Behavioral observations}

During the 3-month experimental period, three twelve-day blocks with behavioral observations on individual mice were scheduled. The $12 \mathrm{MA}$ and the $12 \mathrm{LA}$ mice were identified based on their antenna contacts during the week before the testing.

To a large extent, the focus animals in observation blocks 2 and 3 were identical: in the MA group, nine animals out of 12 were focus animals in both blocks, while in the LA group, there were 10 out of 12 . Block 1 was not further considered in this analysis because it became obvious that the animals had not yet developed their individual activity patterns. Only three of the MA animals in block 1 were also MAs in the following blocks, which made a valid statistical analysis impossible. For this reason, a collective analysis of group differences was done on the animals consistently monitored in both blocks 2 and 3 (see Methods section).

To confirm that the activity patterns of the focus animals were consistent, we ranked all mice by their activity (sum of antenna contacts) over the entire last two months of the experiment and identified the positions of the nine consistent MA and 10 consistent
LA animals within the resulting scope of activities. Eight out of the nine MA animals were above the interquartile range in the overall ranking. Eight out of the $10 \mathrm{LA}$ animals were below the interquartile range. In the overall ranking, the MA animal had twice as many antenna contacts as the LA ones.

Behavioral monitoring was based on an ethogram featuring five broader classes of behavior: Sociopositive behavior (as indicated, e.g., by making body contact), agonistic behavior (e.g., chasing), social exploratory behavior (e.g., approaching or sniffing a conspecific), non-social exploratory behavior (e.g., manipulating an object) and play, and maintenance behavior (e.g., food intake). We did not observe the agonistic behaviors "chase," "bite," "receive bite," or "fight". Therefore, these items were excluded from further analysis. As a positive side result we also found that none of the ENR animals displayed stereotypic behavior, which would be suggestive of stress due to an inappropriate environment (Gross et al., 2012). The category non-social exploratory and play behavior negatively correlated with the total number of antenna contacts ( $r=-0.612, p=0.012$, Pearson correlation). In the combined analysis of blocks 2 and 3, MA mice showed tail tremble more frequently (median $\mathrm{LA}=1.50$, median $\mathrm{MA}=3.00, \quad U=71.0, \quad p=0.033$, Mann-Whitney $U$ test).

We were interested in whether differences in the automatically derived indices of activity, that is, the total number of antenna contacts and RE, correlated with observations from manual behavioral monitoring. To this end, we first tested whether there were any differences in behavioral patterns between LA and MA animals. Statistical comparisons of MA to LA animals for observation blocks 2 and 3 are listed in Table 2 . Second, we tested whether our measure of RE correlated with any of the recorded behaviors. The cumulatively achieved RE scores (cRE at T4) were correlated with single behavior items as well as with the combined categories (results shown in Table 3 ). 
For a more refined statistical analysis, we created five common factor models, each corresponding to one of the five behavioral dimensions in the ethogram. Latent factors are unobserved variables that account for the variation and covariation among a set of variables. Factor analysis yields a more parsimonious representation of the covariation of multivariate observations. By this means, we constructed a single index for each category that best represents the shared variance between all items of a category. In contrast to a simple sum score, this has the added benefit that we can separate measurement error from common variability in the score (Little et al., 1999) and can allow for a differential weighting of the indices. Substituting the individual scales by a single latent factor is effectively a dimensionality reduction that allows us to test our hypotheses in a lower dimensional space, and yields additional information in how far the items of each category are interrelated. In particular, we set up five latent factor models for multivariate count data each assuming a normally distributed latent variable and a log-linear Poisson model for the indicators. All analyses were performed in Mplus 5 (Muthén and Muthén, 1998-2007) using Maximum Likelihood Estimation. Estimates are based on data from the 18 mice whose behavior was observed during both block 2 and block 3. Table 4 lists the estimated factor loadings for each of the five categories.

With regard to the loading patterns we can conclude that all indicators of social exploratory behavior, sociopositive behavior, and non-social exploratory and play behavior contribute to their respective single latent factor. The maintenance factor comprises mainly four out of six dimensions, particularly those related to food intake (drink, feed, root) and resting, and no significant contributions of grooming and nesting. The single factor of agonistic behavior is primarily driven by tail trembling here, which is not a measure of agonistic behavior per se, unless found in the context of an otherwise agonistic interaction. This observation is not surprising as aggressive behaviors were only rarely observed. Overt aggression was not to be expected as the groups consisted solely of females (Schuhr, 1987). Tail trembling presumably here simply reflects apprehension and excitation.

To assess whether LA and MA mice differ in their behavioral factor scores, we tested group differences by estimating a two-group factor model for LA and MA mice and estimating a Wald statistic for the null hypothesis of equivalence on latent factor means across both groups. We found significant differences for nonsocial exploratory and play behavior ( $W=8.868$; $d f=1 ; p=0.003$ ) with MA animals having lower factor scores than LA animals. There were no significant differences for agonistic behavior $(W=2.398$; $d f=1$; $p=0.122)$, maintenance behavior $(W=1.013$; $d f=1$; $p=0.314)$, social exploratory behavior $(W=0.001$; $d f=1 ; \quad p=0.977), \quad$ and socio-positive behavior $(W=0.179 ; d f=1 ; p=0.673)$.

In a second analysis, we correlated the five behavioral factors with RE at corresponding time windows. To this end, we calculated average RE corresponding to the time span of behavioral observation at block 2 and block 3. We ignored days for which protocols noted computer failure. We calculated the correlation of the continuous latent factor and an average RE indicator in a latent variable model (see results in Table 5). We found significant average correlations across both time points for social exploratory $(r=-0.436, p=0.010)$ and nonsocial exploratory and play behavior $(r=-0.446$; $p=0.003)$. Fig. 5 illustrates the correlation of nonsocial exploration and play behavior with RE by plotting estimated factor scores against RE for both observational blocks.

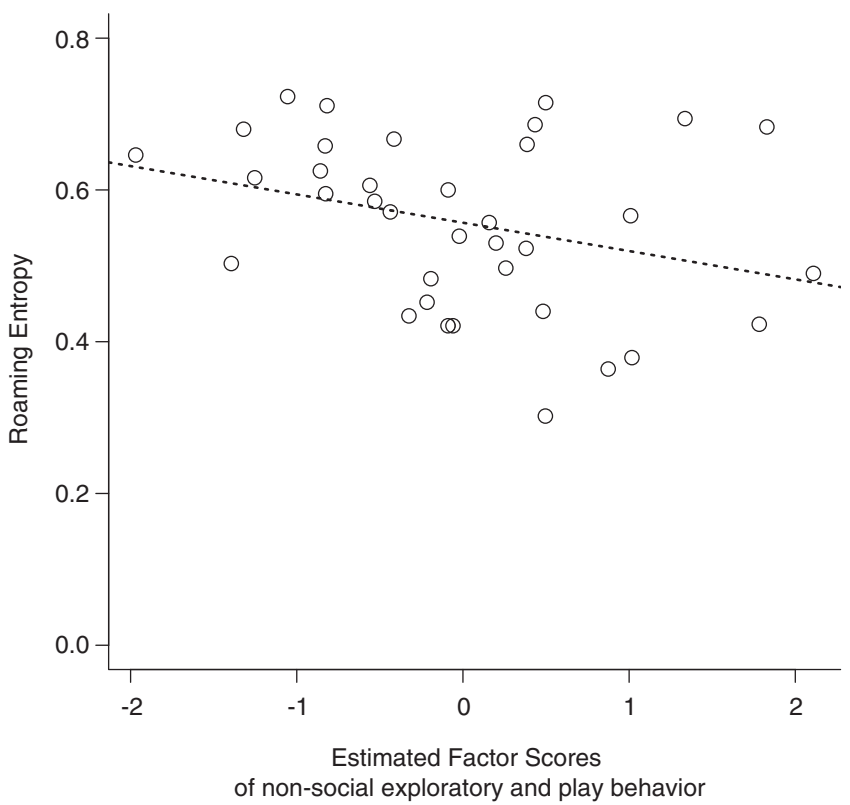

Fig. 5. Estimated factor scores of non-social exploration and play behavior against roaming entropy. Each of the 18 focus animals contributes two points, one corresponding to each observational block. The negative correlation is illustrated by the dashed regression line. 


\section{DISCUSSION}

In the present study we had available two rich dimensions of behavioral monitoring: first, a manual ethological assessment of the MA and LA animals in the enriched environment and, second, data from automatic behavioral tracking. The latter provided a measure of $\mathrm{RE}$ as proxy for areal coverage, novelty seeking and, hence, spatial exploration. The two dimensions are not strongly correlated and cover different aspects of activity.

The rank of a mouse in raw antenna contacts did not predict its $\mathrm{CRE}$, but as CRE had not yet been calculated at the time of the experiment, the mice in our study were selected based on their raw activity. Thus, it turned out that the less active animals by the standard of raw number of antenna contacts were not necessarily the less active mice with respect to their CRE. LA mice were more frequently engaged in self-related behavior and in behavior related to the inanimate environment while MA animals tended to be involved in social behaviors (i.e., behavior related to other mice, agonistic as well as socio-positive) more often in the last observation block. cRE correlated negatively with some items of social behavior. Notably, in our first study, raw antenna contacts did not correlate with adult hippocampal neurogenesis, whereas cRE at the end of the experiment explained $22 \%$ of the variance observed in adult hippocampal neurogenesis among the mice in the enriched environment.

In a factor-analytic approach, we have tested the interrelation of automatically derived indices of activity, that is, RE and live behavioral observation data. We found that non-social exploratory and play behavior was negatively correlated with RE. Animals with high RE indices had fewer counts in items of non-social exploratory and play behaviors. The negative sign of this correlation is not surprising given that non-social exploratory and play behavior items comprise digging, rearing, or object manipulation and thus form a measure of local exploration, while RE reflects a global measure of exploration. The factor analysis also revealed a negative correlation between RE and social exploration behavior. Taken together, mice with high RE values are distinguished as less socially interactive and focused on global rather than local exploration.

These observations might suggest that high levels of adult hippocampal neurogenesis are associated with more individualistic behavioral characteristics, and vice versa. If we accept the idea that the function of new neurons lies in providing flexibility, when new information has to be integrated into pre-established contexts (Dupret et al., 2007; Garthe et al., 2009; Burghardt et al., 2012), a broadly roaming individual might indeed encounter more of such situations than an animal preferring to stay in or near the group. The results of Shemesh et al. (2013) suggest that with the experience of complex environments, the inner-group complexity of behaviors actually decreases.

"Social behavior" includes both socio-positive and agonistic behaviors and in these, the focus animals can play either the acting or the receiving part. There is very little literature on social interactions in exclusively female mouse groups. An entirely female population is, of course, an artificial situation. However, it is a very common one in a laboratory setting. Female social behavior in mixed-sex groups is usually described under the aspect of mating competition and nursing behavior (Weidt et al., 2014), while reports on hierarchical structures and aggression focus primarily on male mice (Mackintosh, 1970; Ralls, 1971; Reynolds, 1971; Desjardins et al., 1973; Haemisch and Gärtner, 1994, 1997; Haemisch et al., 1994; Marashi et al., 2003, 2004; Lewejohann et al., 2009a). We are aware of only a single report on hierarchical structures in groups of female laboratory mice by Schuhr (1987). She demonstrated that subordinate animals in those groups are characterized by frequently seeking contact to the dominant females, while the latter do not get in contact actively. Schuhr noted that hierarchical structures among females are difficult to assess, as they are much subtler than in males and most likely more horizontally organized.

In our ethogram, non-social exploratory and play behavior was combined into a single category. Possibly this arguable decision forfeited the opportunity to establish "play" as independent and informative distinct category with more indicators to be assessed as part of the ethogram. Even in the current configuration, however, the tendency of increased playfulness in LA animals was tangible.

MA animals generally appeared more agitated, as they showed more tail tremble in blocks 2 and 3 (combined analysis). LA mice on the other hand, explored their local environment more (higher amounts of rearing and object manipulation) and were engaged in self-maintenance more often, were more playful and might thus generally be perceived as more relaxed. In a study by Lewejohann et al. (2009a) using the same enclosure as we did with very small differences in the technical setup, dominant male mice were also those with the highest activity levels (i.e., more antenna contacts). These animals needed to constantly patrol and scent-mark the boundaries of their territory, in order to keep their social status, which is in line with previous reports (Mackintosh, 1970; Ralls, 1971; Reynolds, 1971; Desjardins et al., 1973). In contrast to this, in purely female groups, higher levels of activity do not appear to indicate higher rank. In fact, the mere number of antenna contacts did not seem the best parameter to draw conclusions on hierarchies, foreshadowing our cRE-related results.

Stereotypic behaviors represent abnormal behaviors and, for the sake of completeness, were cataloged in the ethogram, which otherwise comprised only normal non-pathological behaviors. Stereotypic behavior is assumed to result from an intrinsic urge to carry out certain innate behaviors serving specific and essential purposes in the wild animal that in a cage cannot be appropriately performed due to spatial constraints and lack of appropriate environmental conditions. In captive animals, stereotypic behaviors are usually considered a sign of stress and often accompanied by social or cognitive deficits (Richter et al., 2009; Gross et al., 2012). Even though the CTR animals had patches of fur 
missing, probably due to over-grooming, none of the large enclosure inhabitants showed anything of this sort, nor were they observed to carry out behaviors classifiable as stereotypical.

ENR mice had accumulated significantly more antenna contacts than CTR animals, which is not altogether surprising, as they had more space and incentive to explore. The RE measure derived from the RFID data goes beyond the mere amount of activity and thus adds a qualitative aspect to the picture of the individual animal's locomotive behavior. Moreover, the cumulatively acquired spatial exploration score, cRE, takes the subtler changes in behavior over time into account. It gives credit to the idea that behavior expresses an inner state at a given time, which is continuously shaped by experience (Freund et al., 2013; Sachser et al., 2013). RE is a measure of areal coverage integrating out the time course of the individual trajectories. Isolating the predictability of where a mouse is at a certain time as measured by RE from the predictability of where a mouse will move to (see Daugherty et al., 2014 for a measure of path complexity associated with brain volume in humans) might provide additional insights into which behavioral patterns are driving developmental processes.

At first glance, it might seem paradoxical that animals initially defined as MAs were not the ones with higher CRE levels (no correlation between total contacts and $\mathrm{CRE}$ ). But CRE and raw antenna contacts illuminate different aspects of activity, as RE includes a qualitative aspect related to areal coverage and spatial preference and thereby "exploration". In our first experiment, high levels of adult neurogenesis were correlated with high cRE. Thus, one could assume that the higher cRE animals of the ENR enclosure reported here would also have more new neurons in the hippocampal dentate gyrus.

If this were the case, the extent of spatial exploration (as quantified by RE) and the gradual broadening of experience within the given space would require more plasticity and therefore be more beneficial to adult neurogenesis than extensive social behaviors. Mice with broader, or precisely more entropic, roaming patterns tend to be more individualistic. The link to adult neurogenesis would not be surprising since the hippocampus is associated with spatial learning. Precursor cells in the dentate gyrus respond to motoric activity (Van Praag et al., 1999; Kronenberg et al., 2003) and their progeny are sensitive to the cognitive challenges of environmental complexity, increasing their survival (Fabel et al., 2009). For the latter, the social context will be relevant. Despite lack of obvious agonistic behavior (fighting over territory etc.) in our experiment, places of preference will have emerged due to avoidance or following of certain other mice (Shemesh et al., 2013). Due to specific social constellations, some mice might have been able to move more freely than others, which in turn could affect the way in which the group used the environment. Thus there is a dire need for further dissection and better describtion the "mix" of possible social, behavioral, and environmental factors contributing to the variance in neurogenesis, preferably by experimentally manipulating the individual factors.
When comparing the ENR group of the present paper to that of the experiment we have reported on previously (Freund et al., 2013), there were differences in CRE variance at the beginning as well as at the endpoint. As we have already briefly discussed in the first paper, it was indeed to be expected that not only the animals would become different, but also that the group as a whole would differ compared to any other group of similar composition. Therefore, across different experimental set-ups no two groups are expected to behave in exactly the same way. Still, the fundamental correlational findings should hold and should be replicable, even though the particular mouse society would be a different one and develop following its own, individual idiosyncratic dynamics.

An early behavioral change in mice was hinted at by the correlations between observational data and CRE values: social exploratory behavior appears to have a positive impact on subsequent $\mathrm{CRE}$ development at an early age (the animals were almost 7 weeks old in observation period 1), while the more individualistic, i.e., self-related behaviors showed an association with higher $\mathrm{cRE}$ values in the later observation blocks. This is also reflected in the greater consistency between observation groups 2 and 3 : the animals seemed to have undergone the greatest change between blocks 1 and 2 . The reason behind this was probably the response to the enrichment for one, but also age-dependent maturation. In line with this is the notion that decline in hippocampal neurogenesis in this age could play a role in the transformation from juvenile and unpredictable ("reckless") behavior to mature and predictable ("cautious") behavior (Altmann, 1974). This theory generally fits with the data presented in Freund et al. (2013): animals with lower levels of neurogenesis have lower cRE values at T4, i.e., they moved in a more predictable way. This would suggest that adult hippocampal neurogenesis retains the brain in a certain "juvenile" reserve, thus providing it with a wider range of behavioral opportunities (Altman et al., 1973; Kempermann, 2008; Amrein and Lipp, 2009; Akers et al., 2014).

Acknowledgments-This study was financed from basic institutional funds. Julia Freund and Imke Kirste were fellows of the International Max Planck Research School on the Life Course (LIFE), Berlin. Mareike Kritzler was supported by the International Research Training Group on 'Semantic Integration of Geospatial Information', funded by Deutsche Forschungsgemeinschaft (DFG).

\section{REFERENCES}

Akers KG, Martinez-Canabal A, Restivo L, Yiu AP, De Cristofaro A, Hsiang H-LL, Wheeler AL, Guskjolen A, Niibori Y, Shoji H, Ohira K, Richards BA, Miyakawa T, Josselyn SA, Frankland PW (2014) Hippocampal neurogenesis regulates forgetting during adulthood and infancy. Science 344:598-602.

Altman J, Brunner RL, Bayer SA (1973) The hippocampus and behavioral maturation. Behav Biol 8:557-596.

Altmann $J$ (1974) Observational study of behavior: sampling methods. Behaviour 49:227-267. 
Amrein I, Lipp H-P (2009) Adult hippocampal neurogenesis of mammals: evolution and life history. Biol Lett 5:141-144.

Burghardt NS, Park EH, Hen R, Fenton AA (2012) Adult-born hippocampal neurons promote cognitive flexibility in mice. Hippocampus.

Daugherty AM, Yuan P, Dahle CL, Bender AR, Yang Y, Raz N (2014) Path complexity in virtual water maze navigation: differential associations with age, sex, and regional brain volume. Cereb Cortex. bhu107.

Desjardins C, Maruniak JA, Bronson FH (1973) Social rank in house mice: differentiation revealed by ultraviolet visualization of urinary marking patterns. Science 182:939-941.

Dupret D, Fabre A, Döbrössy MD, Panatier A, Rodríguez JJ, Lamarque S, Lemaire V, Oliet SHR, Piazza P-V, Abrous DN (2007) Spatial learning depends on both the addition and removal of new hippocampal neurons. PLoS Biol 5:e214.

Fabel K, Wolf SA, Ehninger D, Babu H, Leal-Galicia P, Kempermann G (2009) Additive effects of physical exercise and environmental enrichment on adult hippocampal neurogenesis in mice. Front Neurosci 3:50.

Freund J, Brandmaier AM, Lewejohann L, Kirste I, Kritzler M, Kruger A, Sachser N, Lindenberger U, Kempermann G (2013) Emergence of individuality in genetically identical mice. Science 340:756-759.

Garthe A, Behr J, Kempermann G (2009) Adult-generated hippocampal neurons allow the flexible use of spatially precise learning strategies. PLoS One 4:e5464.

Gross AN, Richter SH, Engel AKJ, Würbel H (2012) Cage-induced stereotypies, perseveration and the effects of environmental enrichment in laboratory mice. Behav Brain Res 234:61-68.

Haemisch A, Gärtner K (1994) The cage design affects intermale aggression in small groups of male laboratory mice: strain specific consequences on social organization, and endocrine activations in two inbred strains (DBA/2J and CBA/J). J Exp Anim Sci 36:101-116.

Haemisch A, Gärtner K (1997) Effects of cage enrichment on territorial aggression and stress physiology in male laboratory mice. Acta Physiol Scand Suppl 640:73-76.

Haemisch A, Voss T, Gärtner K (1994) Effects of environmental enrichment on aggressive behavior, dominance hierarchies, and endocrine states in male DBA/2J mice. Physiol Behav 56:1041-1048.

Kempermann G (2008) The neurogenic reserve hypothesis: what is adult hippocampal neurogenesis good for? Trends Neurosci 31:163-169.

Kritzler M, Lewejohann L, Krüger A, Raubal M, Sachser N (2006) An RFID-based tracking system for laboratory mice in a semi-natural environment. In: IEEE pervasive computing (Spiekermann S, Schmidt A, Gershman A, Michaelles F, eds).
Kronenberg G, Reuter K, Steiner B, Brandt MD, Jessberger S, Yamaguchi M, Kempermann G (2003) Subpopulations of proliferating cells of the adult hippocampus respond differently to physiologic neurogenic stimuli. J Comp Neurol 467:455-463.

Lewejohann L, Hoppmann AM, Kegel P, Kritzler M, Krüger A, Sachser N (2009a) Behavioral phenotyping of a murine model of Alzheimer's disease in a seminaturalistic environment using RFID tracking. Behav Res Methods 41:850-856.

Lewejohann L, Reefmann N, Widmann P, Ambrée O, Herring A, Keyvani K, Paulus W, Sachser N (2009b) Transgenic Alzheimer mice in a semi-naturalistic environment: more plaques, yet not compromised in daily life. Behav Brain Res 201:99-102.

Little TD, Lindenberger U, Nesselroade JR (1999) On selecting indicators for multivariate measurement and modeling with latent variables: when "good" indicators are bad and "bad" indicators are good. Psychol Methods 4:192-211.

Mackintosh JH (1970) Territory formation by laboratory mice. Anim Behav 18:177-183.

Marashi V, Barnekow A, Ossendorf E, Sachser N (2003) Effects of different forms of environmental enrichment on behavioral, endocrinological, and immunological parameters in male mice. Horm Behav 43:281-292.

Marashi V, Barnekow A, Sachser N (2004) Effects of environmental enrichment on males of a docile inbred strain of mice. Physiol Behav 82:765-776.

Muthén LK, Muthén BO (1998) Mplus user's guide. fifth ed. Los Angeles, CA: Muthén \& Muthén.

Ralls K (1971) Mammalian scent marking. Science 171:443-449.

Reynolds E (1971) Urination as a social response in mice. Nature 234:481-483.

Richter SH, Garner JP, Würbel H (2009) Environmental standardization: cure or cause of poor reproducibility in animal experiments? Nat Methods 6:257-261.

Sachser N, Kaiser S, Hennessy MB (2013) Behavioural profiles are shaped by social experience: when, how and why. Philos Trans R Soc Lond B Biol Sci 368. 20120344-20120344.

Schuhr B (1987) Social structure and plasma corticosterone level in female albino mice. Physiol Behav 40:689-693.

Shemesh Y, Sztainberg Y, Forkosh O, Shlapobersky T, Chen A, Schneidman E (2013) High-order social interactions in groups of mice. eLife 2. e00759-e00759.

Terranova ML, Laviola G (2005) Scoring of social interactions and play in mice during adolescence. Curr Protoc Toxicol. 13.10.11.

Van Praag H, Kempermann G, Gage FH (1999) Running increases cell proliferation and neurogenesis in the adult mouse dentate gyrus. Nat Neurosci 2:266-270.

Weidt A, Lindholm AK, König B (2014) Communal nursing in wild house mice is not a by-product of group living: females choose. Naturwissenschaften 101:73-76. 